\title{
Hydrothermal Synthesis of Multi-Color Fluorescent Carbon Dots from Sappan Wood (Caesalpinia sappan) as an Acid-Base Indicator
}

\author{
ADIN HASRUDIN ${ }^{1}$, ZAENAL ABIDIN ${ }^{*}$, NURUL HIEDAYATI ${ }^{2}$, VICKY PRAJAPUTRA ${ }^{1,3}$ \\ ${ }^{1}$ IPB University, Faculty of Mathematic and Natural Sciences, Department of Chemistry, Bogor, Indonesia \\ ${ }^{2}$ Syarif Hidayatullah State Islamic University, Faculty of Medicine, Department of Pharmacy, Jakarta, Indonesia \\ ${ }^{3}$ IPB University, Study Program of Natural Resources and Environmental Management, Bogor, Indonesia
}

\begin{abstract}
Caesalpinia sappan L., known as sappan wood, has been extensively explored by many researchers because it contains various structural types of phenolic compounds. This study objectives are to investigate the effect of $\mathrm{pH}$ on the flourescent color of carbon dots (CDs) from sappan wood and its application as an acid-base indicator under UV light for the first time. CDs were synthesized through the hydrothermal route in ethanol solvents. The synthesized CDs were further characterized by UV-Vis spectrophotometry, fluorescence and Fourier Transform Infrared (FT-IR) spectroscopy. Based on the results, CD solutions performed multiple flourescent colors (yellow, orange and green) under UV light at $365 \mathrm{~nm}$ when the $\mathrm{pH}$ was adjusted to be 1, 3, 5, 7, 9 and 11. These multiple colors were produced because of the instability of phenolic compounds in the sample, especially brazilin which was easily oxidized to brazilein in alkaline condition. According to the FT-IR spectra, the most prominent differences of the sample before and after the treatment process was observed in the peak region ranging from 1500-1700 $\mathrm{cm}^{-1}$. The characteristic band of carbonyl groups was identified at $1697 \mathrm{~cm}^{-1}$ in the treated sample ( $\mathrm{pH} 9$ ), but it was unobserved in the spectrum of sappan wood and the treated sample ( $\mathrm{pH}$ 5). The presence of carbonyl group can be assumed that brazilin has been oxidized to brazilein after the alkaline treatment process and making the flourescent color was gradually shifted from yellow to green. Under normal light, the sappan wood's extract and CD solution produced yellow and red colors in acidic and alkaline conditions, respectively. However, only CD solutions which have yellow, orange and green emissions in different $\mathrm{pH}$ conditions under UV light. Therefore, the synthesized CDs can be used as an acid-base indicator in both different light conditions.
\end{abstract}

Keywords: absorption, brazilin, brazilein, emission, materials

\section{Introduction}

Nowadays, carbon dots (CDs) have attracted widespread attention as one of the most important material for a variety of applications because of its low toxicity, good water soluble, chemical inertness and more environmentally friendly [1]. They also exhibit different optical properties depending on their size, such as chemiluminescence, photoluminescence and photoinduced electron transfer. These desirable properties making CDs are well applicable in fluorescence imaging, chemical-sensing, bio-sensing and drug delivery [2].

Several synthetic processes used to convert the precursor to be CDs are ultrasonication, solvothermal, chemical oxidation and hydrothermal [3]. Among of these conventional method, hydrothermal is the most frequently method used because of its simple process, environmentally friendly and higher yield with a good CD surface in fluorescence properties. Many researchers use various chemical precursors containing $\mathrm{N}$ and $\mathrm{S}$ atoms, such as citric acid, citric acid-urea and thiourea as the source of CDs [4]. Although the produced CD using these precursors has good color intensity, it still requires expensive costs to prepare $\mathrm{CDs}$ on a large scale. Therefore, the use of natural sources for preparing CDs are investigated by experts [5].

\footnotetext{
*email: abidinzed@apps.ipb.ac.id
} 
The previous research which conducted by Chen et al. [6] have been reported the use of garlic to synthesis CDs using hydrothermal treatment, showing it could be an alternative as a carbon source. Besides garlic, other natural sources that received attention came mainly from widely available plants such as sappan wood (Caesalpinia sappan L.). Sappan wood contains water-soluble flavonoids namely brazilin, protosappanin and haematoxylin. Brazilin is the main flavonoid in sappan wood as a yellow dye with a $\mathrm{C}_{16} \mathrm{H}_{14} \mathrm{O}_{5}$ structure in crystalline form which has a melting point of $145-149^{\circ} \mathrm{C}$ and a moleculer weight of $286 \mathrm{~m} / \mathrm{z}$. However, brazilin will be brazilein as an oxidized form with a red color when dissolved in an ethanol solvent because of its unstability in alkaline $p \mathrm{H}$ [7]. The differences color in acidic and alkaline condition making this plant can be applied as an acid-base indicator.

In this work, sappan wood was used as a natural source for the first time to synthesis CDs by using simple hydrothermal treatment. The effect of $p \mathrm{H}$ on the fluorescence color after the synthesis process will be evaluated. The produced CDs will also be tested as an acid-base indicator under visible and UV lights.

\section{Materials and methods}

\subsection{Materials}

Sappan woods as starting material were obtained from IPB University's laboratory. The sample was mashed and dried for 2 days to remove moisture. The dried sample was separately extracted using two different solvents (distilled water and $96 \%$ ethanol) by maceration method. All chemicals used including ethanol $96 \%$, hydrochloric acid $(\mathrm{HCl})$ and sodium hydroxide $(\mathrm{NaOH})$ were purchased from inorganic chemistry laboratory of IPB University.

\subsection{Synthesis of multi-color fluorescent CDs}

CDs were synthesized by dissolving 2 grams of dried samples into $25 \mathrm{~mL}$ of $96 \%$ ethanol under stirring condition for $30 \mathrm{~min}$. The mixture was transfered and heated in a closed stainless steel autoclave at $150^{\circ} \mathrm{C}$ for $8 \mathrm{~h}$, as represented in Scheme 1. Then, the product was allowed to cool naturally at room temperature and centrifuged at $4500 \mathrm{rpm}$ for $15 \mathrm{~min}$. The pure of CD solution was obtained after the supernatant was filtered using a Whatman filter paper.

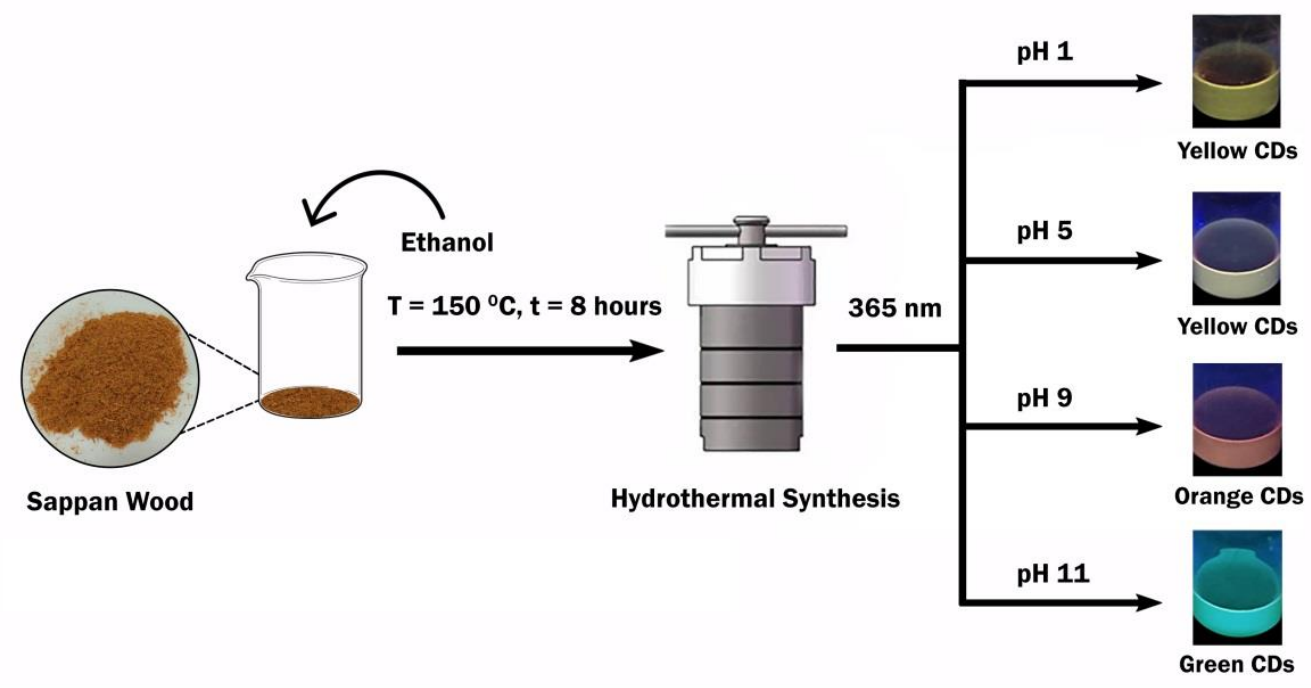

Scheme 1. Schematic illustration of the synthesis of CDs

\subsection{Characterization of CDs}

After the synthesis process, the $p \mathrm{H}$ of $\mathrm{CD}$ solutions was adjusted to be 1, 3, 5, 7, 9 and 11, which controlled using $0.1 \mathrm{M} \mathrm{HCl}$ or $\mathrm{NaOH}$. The presence of $\mathrm{CDs}$ was revealed by irradiating these solutions under a UV lamp (365 nm) (Scheme 1, right). Then, the fluorescent intensity was measured by a fluorescence spectrophotometer. To get the solid phase, $1 \mathrm{~mL}$ of CD solutions ( $p \mathrm{H} 5$ and 9) was dried 
at $100{ }^{\circ} \mathrm{C}$ and characterized by FTIR spectroscopy using a Perkin Elmer Spectrum One spectrometer, which recorded from $500-4,000 \mathrm{~cm}^{-1}$. The FTIR spectra before and after the treatment process were compared.

\subsection{Aplication of CDs as an acid-base indicator}

About $0.5 \mathrm{~mL}$ of the sappan wood's extract and $\mathrm{CD}$ solution $(p \mathrm{H} 5)$ was transfered separately to the four vials containing $4 \mathrm{~mL}$ of distilled water. The $p \mathrm{H}$ of each solution was controlled to be 5, 6, 9 and 11, respectively. Then, all vials were illuminated under a UV light at $365 \mathrm{~nm}$ for a minute. The maximum wavelength of solutions was determined using a UV-Vis spectrophotometer.

\section{Results and discussions}

\subsection{Effect of $\mathrm{pH}$ on the synthesized CDs from sappan wood}

Sappan wood contains various structural types of phenolic compounds, such as coumarin $\left(\mathrm{C}_{9} \mathrm{H}_{6} \mathrm{O}_{2}\right)$, xanthone $\left(\mathrm{C}_{13} \mathrm{H}_{8} \mathrm{O}_{2}\right)$, chalcone $\left(\mathrm{C}_{15} \mathrm{H}_{12} \mathrm{O}_{5}\right)$ and brazilin $\left(\mathrm{C}_{16} \mathrm{H}_{14} \mathrm{O}_{5}\right)$. Among these phenolic compounds, brazilin has been reported as the major component in sappan wood, which produced yellow color in solution and more stable under the acidic $p \mathrm{H}$ [7]. The synthesis of CDs from sappan wood as starting material was carried out through the hydrothermal treatment using ethanol solvent. Ethanol is well-known as a good solvent for extracting all flavonoid compounds, including brazilin because of the same polarity [8]. Therefore, ethanol solvent was selected in this work.

The solution produced after the synthesis process was in red color $(p \mathrm{H} 5)$. It was caused due to the use of ethanol solvent $(p \mathrm{H} \mathrm{8})$ in the treatment process making the solution to be alkaline. Brazilin is unstable under alkaline $p \mathrm{H}$ and will be brazilein as an oxidized form, which appears in red color [9]. Thus, brazilein was formed when the hydrothermal process is complete. To study the effect of $\mathrm{pH}$ on the fluorescent color, the $p \mathrm{H}$ of $\mathrm{CD}$ solutions was adjusted to be $1,3,5,7,9$ and 11 . In this work, we only used a simple technique to prove the successful of $\mathrm{CD}$ formation by irradiating the $\mathrm{CD}$ solution under a UV lamp at $365 \mathrm{~nm}$. The fluorescent color of solution indicates the presence of CDs. Figure 1 shows the color of CD solutions under both visible and UV lamps with ethanol as the control solution at room temperature. As seen, acid caused brazilein to become orange color and alkaline produced a deep crimson color in solution under normal light. The fluorescent colors of yellow $(p \mathrm{H} 1,3,5)$, orange $(p \mathrm{H} \mathrm{7,9)}$ and green $(p \mathrm{H} 11)$ were obtained by illuminating CD solutions below a UV lamp at $365 \mathrm{~nm}$. According to the previous report, natural plants containing several organic molecules including cellulose, glucose and phenolic compounds are recommended for the synthesis of CDs [1012]. This is because the large number of carbon-containing small molecule in natural plants can be polymerized and carbonized to form a carbon backbone at high temperature condition, which can further form fluorescent CDs. The formation mechanism of synthesized CDs has been explained in detail by Liu et al. [13].

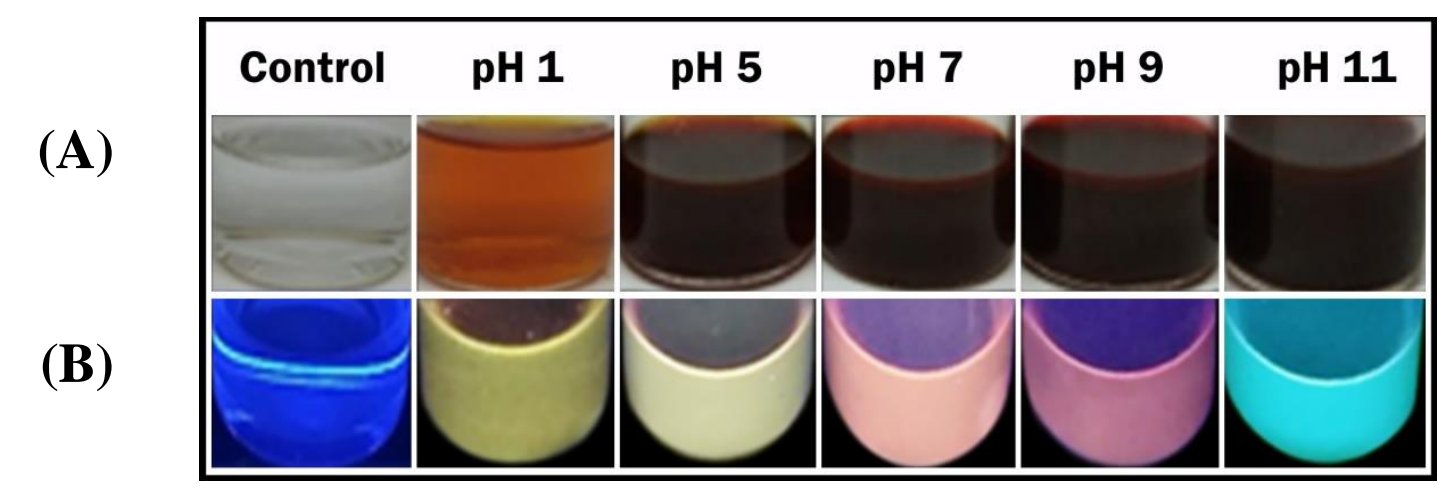

Figure 1. Multi-color fluorescent $\mathrm{CDs}$ in different $p \mathrm{H}$ conditions under visible (A) and UV light (B) 
Each flourescent color has a different energy level, depending on the excitation distance (band gap energy) between the valence and conduction bands [14]. The fluorescent intensity of CDs was measured by using a fluorescence spectrophotometer in each $p \mathrm{H}$ condition. Figure 2 shows the

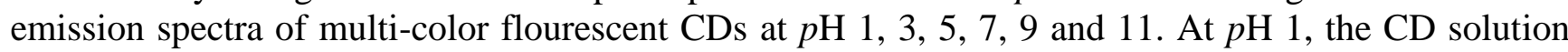
emits yellow color in the wavelength of $570 \mathrm{~nm}$. The emission peak was gradually shifted from 570 $\mathrm{nm}$ (yellow) to $600 \mathrm{~nm}$ (orange) at $\mathrm{pH}$ 7. Two emission peaks which appeared in area $470 \mathrm{~nm}$ and 600 $\mathrm{nm}$ were observed in dark orange color at $p \mathrm{H}$ 9. When the $p \mathrm{H}$ solution was adjusted to be 11 , the solution contributes a green color in the wavelength of $500 \mathrm{~nm}$. The photoluminescence (PL) spectrum of sappan wood extract is not shown due to the absence of fluorescence in solution under a UV lamp.

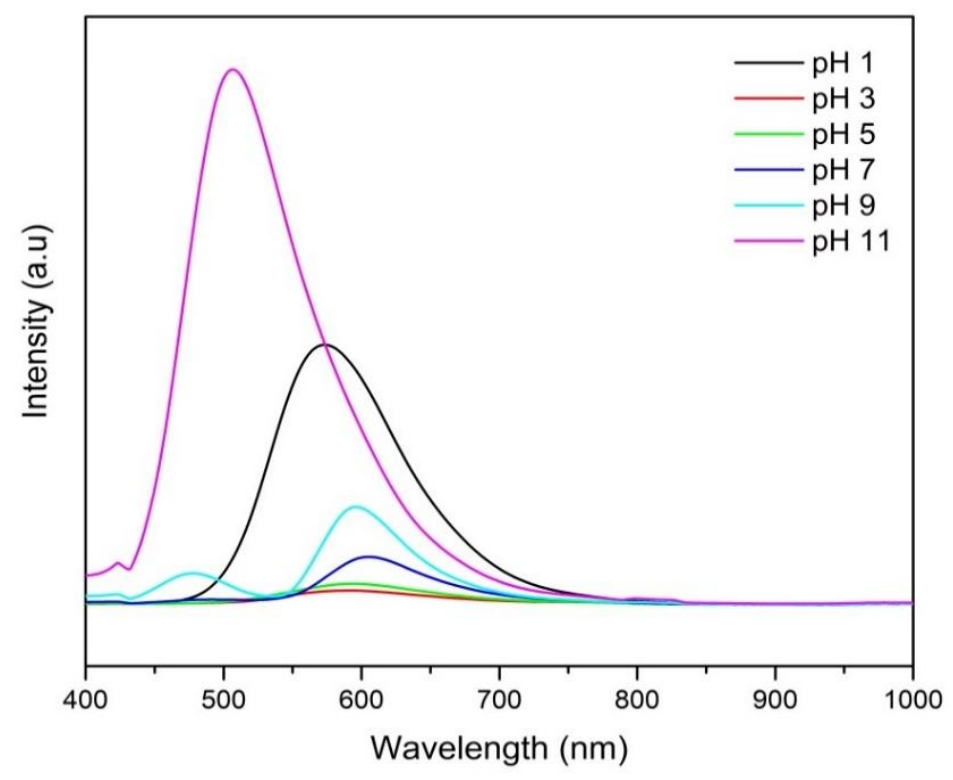

Figure 2. The emission spectra of CDs in ethanol solvents

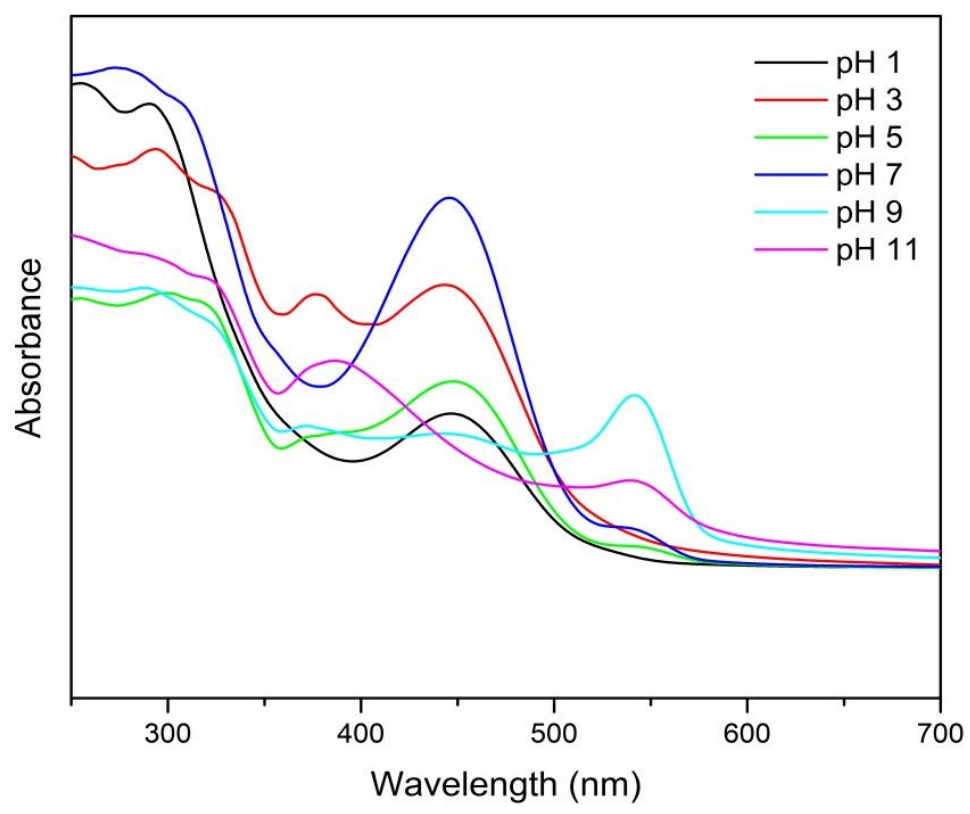

Figure 3. The absorption spectra of CDs in ethanol solvents

Figure 3 overlays the absorption spectra of $\mathrm{CD}$ solutions under acidic and alkaline $p \mathrm{H}$ conditions measured by UV-Vis spectrophotmeter. The presence of brazilin was detected in the $p \mathrm{H} 1$ and 3 at the wavelength of $290 \mathrm{~nm}$. The similar result is also obtained by Nirmal et al, which reported the 
maximum absorption of brazilin at $292 \mathrm{~nm}$ [7]. The maximum wavelength of $450 \mathrm{~nm}$ at $p \mathrm{H} \mathrm{1,3,5}$ and 7 was corresponded as the spectra of brazilien. However, the spectra was shifted from $450 \mathrm{~nm}$ to 540 $\mathrm{nm}$ at $\mathrm{pH} 9$ and 11. Therefore, it can be inferred that brazilein has two maximum absorption wavelengths at $450 \mathrm{~nm}$ and $540 \mathrm{~nm}$.

\subsection{FT-IR spectra of samples}

CDs were characterized by FT-IR spectroscopy to identify the functional group in each sampel under acidic and alkaline $p \mathrm{H}$. Figure 4 presents the FT-IR spectra of samples before and after the hydrothermal treatment. Before the treatment process, there are several peaks occured, especially at $1040 \mathrm{~cm}^{-1}, 1455 \mathrm{~cm}^{-1}, 1507 \mathrm{~cm}^{-1}, 1605 \mathrm{~cm}^{-1}$, and $3200 \mathrm{~cm}^{-1}$. The peak at $1040 \mathrm{~cm}^{-1}$ was assigned to the ring stretching of phenolic compounds. In the region between $1450 \mathrm{~cm}^{-1}$ and $1610 \mathrm{~cm}^{-1}$, the vibration peaks of $\mathrm{C}=\mathrm{C}$ were obtained. Generally, the FTIR spectra before and after the treament process are similar. One of the most prominent differences of these samples were found in the vibration peak at $1697 \mathrm{~cm}-1(p \mathrm{H} 9)$, which showed the presence of carbonyl $(\mathrm{C}=\mathrm{O})$ groups. This carbonyl group indicated the formation of brazilein in $\mathrm{CDs}(p \mathrm{H} 9)$ and was not appeared in the spectrum of sappan wood or $\mathrm{CDs}(p \mathrm{H} \mathrm{5})$ samples. Another study has also reported that brazilein spectrum exhibits a strong band at $1697 \mathrm{~cm}^{-1}[15]$.

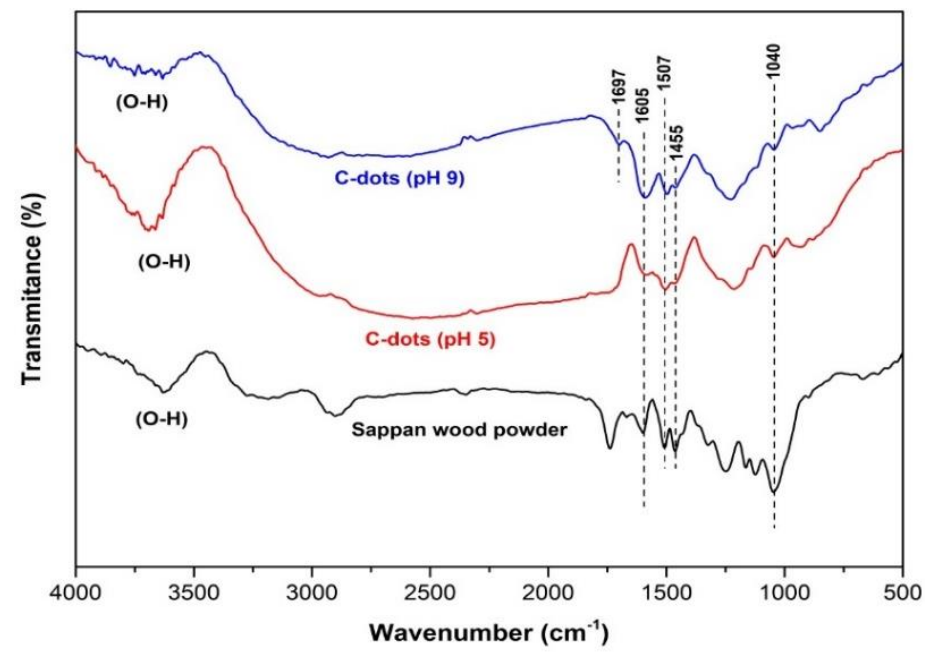

Figure 4. The FT-IR spectrum of samples before and after the treatment process

\subsection{CDs as an acid-base indicator}

Sappan wood's extract has been widely applied as an acid-base indicator, but its application as an indicator is only used under visible light. Therefore, we investigated the use of synthesized CDs from sappan wood as an acid-base indicator below a UV light for the first time. Figure 5 and 6 are shown the color of sappan wood's extract and CDs in various $p \mathrm{H}$ conditions under visible and UV lights, respectively.

(A)

(B)

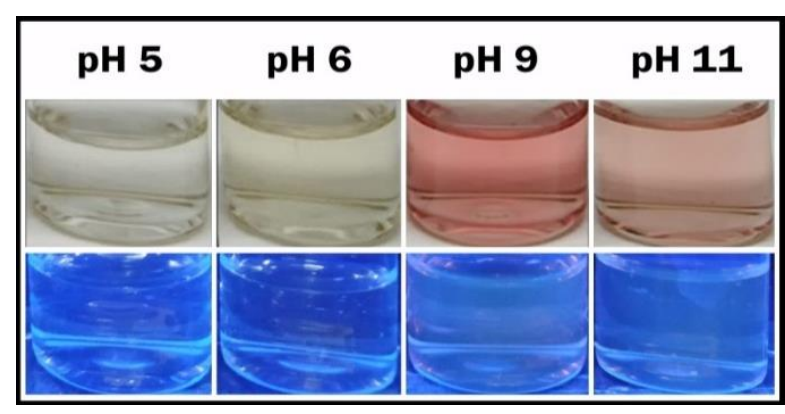

Figure 5. The color of sappan wood solution under normal (A) and UV (B) lights 


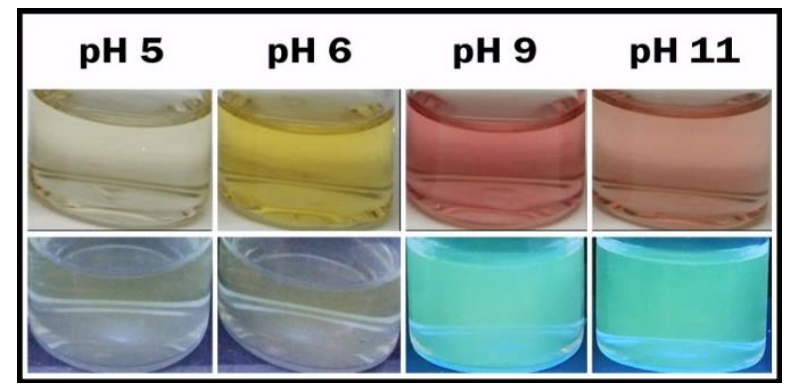

Figure 6. The color of CDs under normal (A) and UV (B) lights

The sappan wood and CD solutions have slightly yellow an red colors in different $p \mathrm{H}$ conditions under normal light. Figure 7 represents the absorption spectra of each sample. Based on the result, brazilien absorption peak in the wavelength around 540 was showed under alkaline $p \mathrm{H}$. However, only sappan wood did not show fluorescent color under UV lamp. The synthesized CDs from sappan wood produced yellow color in acidic $p \mathrm{H}$ and green color in alkaline $p \mathrm{H}$. These results revealed that CDs can be applied as acid-base indicator under both light conditions. Besides, the color of fluorescent formed is reversible in both acidic and alkaline $p \mathrm{H}$ because it has the similar color when the $p \mathrm{H}$ was adjusted as before. The use of CDs from sappan wood can also be referenced to develop a biosensor in medicine field.
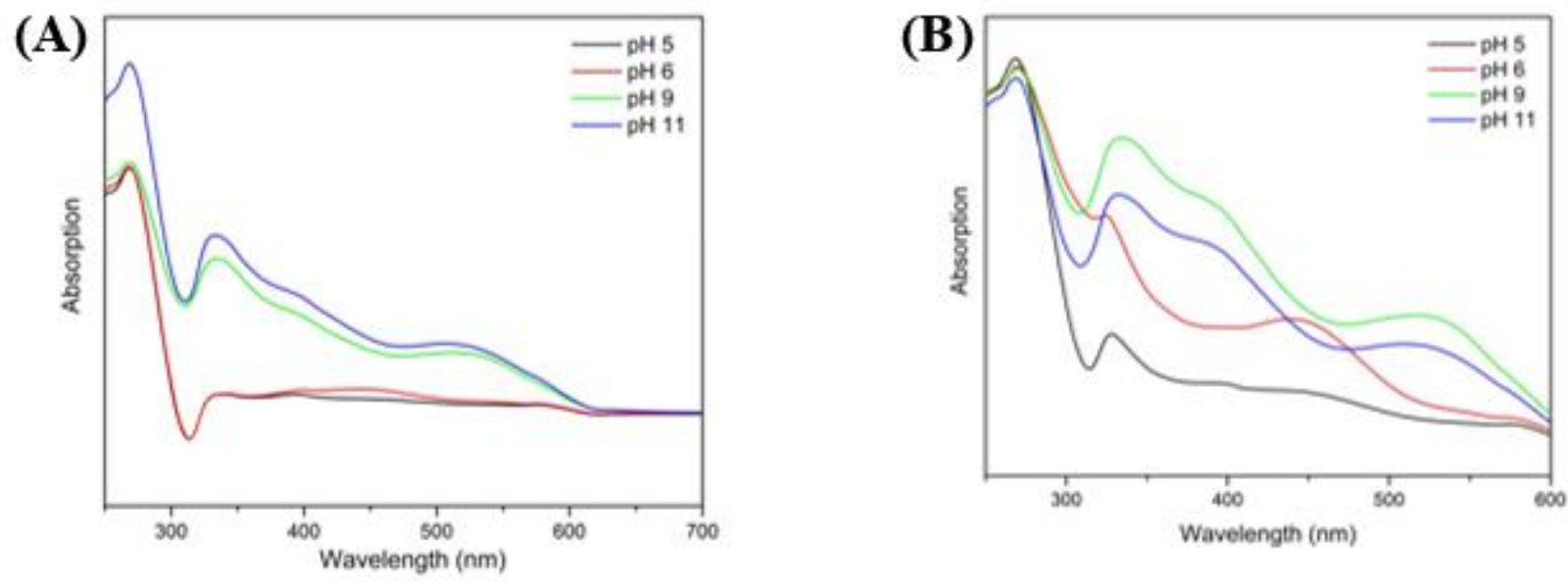

Figure 7. The absorption spectra of sappan wood (A) and CD (B) solutions

\section{Conclusions}

The multi-color fluorescent CDs were successfully synthesized through simple hydrothermal method using ethanol solvent under various $p \mathrm{H}$ condition. $\mathrm{CD}$ solutions exhibited yellow, orange and green fluorescent at $p \mathrm{H} \mathrm{5,9}$ and 11, respectively. The fluorescent intensity was measured by fluorescence spectrophotometer. At $p \mathrm{H} 1$ and 3, the maximum wavelength of brazilin was detected at $290 \mathrm{~nm}$. When the $p \mathrm{H}$ of solution increased, the brazilin peak was disappeared due to the formation of brazilein. Brazilein has two maximum wavelengths at 450 and $540 \mathrm{~nm}$. The FT-IR spectra before and after the hydrothermal treatment were used in order to identify the functional groups in the sample. The results showed that brazilin was obtained in the sample of sappan wood and $\mathrm{CD}(p \mathrm{H} 5)$ because of the absence of carbonyl groups. The sappan wood and CD solution have the similar color under normal light. However, only the synthesized CDs from sappan wood produced multi-color fluorescent under UV light at $360 \mathrm{~nm}$. Therefore, CDs can be used as an acid-base indicator in both light conditions. 
Acknowledgments: The authors deeply thank Department of Chemistry (Inorganic laboratory), IPB University for supporting and facilitating this research with the necessary equipment.

\section{References}

1. SHARMA, A., DAS, J., Small molecules derived carbon dots: synthesis and applications in sensing, catalysis, imaging, and biomedicine, J Nanobiotechnol, 17(1), 2019, 92.

2. ZHENG, Y., XIE, G., ZHANG, X., CHEN, Z., CAI, Y., YU, W., LIU, H., SHAN, J., LI, R., LIU, Y., LEI, B., Bioimaging Application and Growth-Promoting Behavior of Carbon Dots from Pollen on Hydroponically Cultivated Rome Lettuce, ACS Omega, 2(7), 2017, 3958-3965.

3. WANG, X., FENG, Y., DONG, PEIPEI., HUANG, J., A Mini Review on Carbon Quantum Dots: Preparation, Properties, and Electrocatalytic Application, Front. Chem, 7, 2019, 671.

4. DONG, Y., PANG, H., YANG, H. B., GUO, C., SHAO, Y., CHI, T., Carbon-based dots co-doped with nitrogen and sulfur for high quantum yield and excitation-independent emission, Angew Chem Int Ed, 52, 2013, 7800-7804.

5. ZHANG, X., JIANG, M., NIU, N., CHEN , Z., LI, S., LIU, S., LI, J., Natural-Product-Derived Carbon Dots: From Natural Products to Functional Material, Chem. Sus. Chem, 11(1), 2017, 11-24.

6. CHEN, Y., WU, Y., WENG, B., WANG, B., LI, C., Facile synthesis of nitrogen and sulfur codoped carbon dots and application for Fe(III) ions detection and cell imaging, Sensor and Actuators B: Chemical, 223, 2016, 689-696.

7. NIRMAL, N. P., RAJPUT, M. S., PRASAD, R.G.S.V., AHMAD, M., Brazilin from Caesalpinia sappan heartwood and its pharmacological activities: A review, Asian Pac J Trop Med, 8(6), 2015, 421-430.

8. WANG, H., HELLIWELL, K., Determination of flavonols in green and black tea leaves and green tea infusions by high-performance liquid chromatography, Food Res Int, 34(2-3), 2011, 223-227.

9. DAPSON, R., BAIN, C., Brazilwood, sappanwood brazilin and the red dye brazilein: from textile dyeing and folk medicine to biological staining and musical instruments, Biotechnic \& Histochemistry, 90(6), 2015, 401-423.

10. LIU, M. L., CHEN, B. B., LI, R. S., LI, C. M., ZOU, H. Y., HUANG C. Z., Dendritic CuSe with hierarchical side-branches: Synthesis, efficient adsorption, and enhanced photocatalytic activities under daylight, ACS Sustainable Chem Eng, 5, 2017, 4154-4160.

11. CHEN, B. B., LIU, H., HUANG, C. Z., LING, J., WANG J., Rapid and convenient synthesis of stable silver nanoparticle with kiwi juice and its novel application for detecting protease K, New J. Chem, 39, 2015, 1295-1300.

12. LI, R. S., LIU, H., CHEN, B. B., ZHANG, H. Z., HUANG C. Z., WANG, J., Stable gold nanoparticle as a novel peroxidase mimic for colorimetric detection of cysteine, Anal. Methods, 8 , 2016, 2494-2501.

13. LIU, M. L., CHEN, B. B., LI, C. M., HUANG, C. Z., Carbon dots: synthesis, formation mechanism, fluorescence origin and sensing applications, Green Chem, 21, 2019, 449-471.

14. ROY, P., CHEN, P. C., PERIASAMY, A. P., CHEN, Y. N., CHANG, H. T., Photoluminescent carbon nanodots: synthesis, physicochemical properties and analytical applications, Material Today, 18(8), 2015, 447-458.

15. DE OLIVEIRA, L. F. C., EDWARS, H. G. M., VELOZO, E. S., NESBITT, M., Vibrational spectroscopic study of brazilin and brazilein, the main constituents of brazilwood from Brazil, Vibrational Spectroscopy, 28, 2002, 243-249.

Manuscript received: 10.04 .2020 